\title{
Scintillation and Optical Properties of Ce-Doped Fluoride Glass Samples with Different Ce Concentrations
}

\author{
Kohei Yamanoi*, Takahiro Murata ${ }^{1}$, Takayuki Yanagida ${ }^{2}$, Yutaka Fujimoto ${ }^{3}$, \\ Melvin John F. Empizo, Keisuke Iwano, Yuki Iwasa, Ren Arita, \\ Yuki Minami, Yasunobu Arikawa, Toshihiko Shimizu, Nobuhiko Sarukura, \\ Takayoshi Norimatsu, Hiroshi Azechi, Shigeru Fujino ${ }^{4}$, \\ Hideki Yoshida ${ }^{5}$, Nakahiro Satoh ${ }^{5}$ and Hirofumi Kan ${ }^{6}$ \\ Institute of Laser Engineering, Osaka University, 2-6 Yamadaoka, Suita, Osaka 565-0871, Japan \\ ${ }^{1}$ Kumamoto University, 2-40-1 Kurokami, Kumamoto 860-8555, Japan \\ ${ }^{2}$ Nara Institute of Science and Technology, 8916-5 Takayama, Ikoma, Nara 630-0192, Japan \\ ${ }^{3}$ Kyushu Institute of Technology, Graduate School of Life Science \& Systems Engineering, \\ 2-4 Hibikino, Wakamatsu-ku, Kitakyushu, Fukuoka 808-0196, Japan \\ ${ }^{4}$ Kyushu University, Kyushu University, 6-1 Kasuga, Fukuoka 816-8580, Japan \\ ${ }^{5}$ Ceramic Research Center of Nagasaki, \\ 605-2 Hiekoba, Hasami, Higashisonogi, Nagasaki 859-3726, Japan \\ ${ }^{6}$ Hamamatsu Photonics K.K., 1-8-3 Shinmiyakoda, Kitaku, Hamamatsu 431-2103, Japan
}

(Received October 10, 2014; accepted February 2, 2015)

Key words: cerium, glass, scintillation, photoluminescence

Scintillation and optical properties of cerium (Ce)-doped $20 \mathrm{Al}\left(\mathrm{PO}_{3}\right)_{3}-80 \mathrm{LiF}$ (APLF) glass samples prepared by melt quenching are investigated. The properties of these samples are dependent on the doping concentration, and quenching with high $\mathrm{Ce}$ concentration is not observed. A high quantum efficiency of $95 \%$ is achieved with 3 mol\% Ce and with a decay time of around $40 \mathrm{~ns}$ from the allowed $5 \mathrm{~d}-4 \mathrm{f}$ transition at $350 \mathrm{~nm}$. This quantum efficiency is high compared to other Ce-doped materials such as $\mathrm{Ce}: \mathrm{LiCaAlF}_{6}$ laser crystals. Ce-doped APLF glass can then be used as illuminators, wavelength converters, and scintillators in the ultraviolet region.

\section{Introduction}

Cerium (Ce)-doped optical materials have been developed throughout the years as scintillators, radiation detectors, and laser materials. ${ }^{(1-3)}$ For inertial confinement fusion (ICF), 20Al $\left(\mathrm{PO}_{3}\right)_{3}-80 \mathrm{LiF}$ (APLF) glass samples doped with $\mathrm{Ce}$, praseodymium ( $\mathrm{Pr}$ ), neodymium $(\mathrm{Nd})$, and erbium $(\mathrm{Er})$ have also been investigated for neutron detector

*Corresponding author: e-mail: yamanoi-k@ile.osaka-u.ac.jp 
applications. $^{(4,5)}$ These APLF samples are sensitive to neutrons because of their high concentration of lithium ( $\left.{ }^{6} \mathrm{Li}\right)$. APLF glass has a fast response without afterglow compared to conventional Li glass neutron scintillators such as GS2 (Saint-Gobain). ${ }^{(6,7)}$ APLFs have also high transmittance from $160 \mathrm{~nm}$ to the ultraviolet (UV) region. With these characteristics, APLF glasses are prominent candidates for down-scattered neutron detection for ICF experiments. The detailed research and improvement of these materials are therefore necessary. In a previous paper, the scintillation properties of $1 \mathrm{~mol} \% \mathrm{Ce}$ doped APLF glass are reported. ${ }^{(8)}$ In this present paper, we report the scintillation and photoluminescence properties of Ce-doped APLF glass samples with different doping concentrations ranging from 0.1 to $3 \mathrm{~mol} \%$.

\section{Materials and Experiments}

Ce-doped APLF glass samples, hereafter referred to as APLF80+Ce, were prepared by melt quenching. ${ }^{(9)}$ The chemical composition of each sample was $20 \mathrm{Al}\left(\mathrm{PO}_{3}\right)_{3}$ $80 \mathrm{LiF}+x \mathrm{CeF}_{3}$, where $x$ is $0.1,0.3,0.5,1,2$, and $3 \mathrm{~mol} \%$. A $12 \mathrm{~g}$ mixture of high-purity aluminum phosphite $\left[\mathrm{Al}\left(\mathrm{PO}_{3}\right)_{3}\right]$, lithium fluoride $(\mathrm{LiF})$, and cerium fluoride $\left(\mathrm{CeF}_{3}\right)$ was melted in a glassy carbon crucible at $1000{ }^{\circ} \mathrm{C}$ for $0.5 \mathrm{~h}$ under nitrogen atmosphere. $\mathrm{Al}\left(\mathrm{PO}_{3}\right)_{3}$ was eventually added to avoid the immediate crystallization of LiF. The glass melt was then cooled down and subsequently annealed at $400{ }^{\circ} \mathrm{C}$ near the glass transition temperature. All samples were then cut into $23 \mathrm{~mm}$ diameter $(\phi) \times 8 \mathrm{~mm}$ thickness $(t)$ disks and were polished for optical and scintillation measurements.

The photoluminescence (PL), PL excitation (PLE) spectrum, and absolute quantum yield were measured using an absolute PL quantum yield measurement system (Quantaurus-QY, Hamamatsu Photonics). The absolute quantum yield (QY) was calculated by the expression:

$$
Q Y=\frac{N_{\text {emit }}}{N_{\text {absorb }}}
$$

where $N_{\text {emit }}$ and $N_{\text {absorb }}$ are the numbers of emitted and absorbed photons, respectively. The X-ray induced radioluminescence spectra were measured using an X-ray generator and a charge-coupled device (CCD)-based spectrometer (DU-420-BU2, Andor). The geometry of the system is described in detail in ref. 10. The supplied bias voltage and tube current of the X-ray generator were set at $80 \mathrm{kV}$ and $2 \mathrm{~mA}$, respectively. The absolute quantum efficiency, on the other hand, was evaluated using the measured pulse height distribution. The APLF sample was wrapped with Teflon tape for the collection of scintillated photons and was then coupled to a photomultiplier tube (PMT) (R7600-200, Hamamatsu Photonics) using an optical grease. A californium $252\left({ }^{252} \mathrm{Cf}\right)$ pellet was used as the neutron source. $5 \mathrm{~cm}$ thick lead $(\mathrm{Pb})$ blocks were placed between the ${ }^{252} \mathrm{Cf}$ and the APLF sample in order to cut the background gamma $(\gamma)$ rays from the ${ }^{252} \mathrm{Cf}$. The PMT was then supplied with $-700 \mathrm{~V}$, and the signals were read out from the PMT anode. Upon detection of a neutron from the ${ }^{252} \mathrm{Cf}$, the signals were fed into a preamplifier (ORTEC 113 ) and then to a shaping amplifier (ORTEC 572) with $2 \mu$ s shaping time. The signals 
were digitally converted by a multichannel analyzer (Pocket MCA 8000A, Amptek) and were recorded by a personal computer. The absolute quantum yield was calibrated in comparison to that of a conventional scintillator, GS20 with 7000 photons. ${ }^{(6,7)}$ The decay time measurements were also carried out using a digital oscilloscope (TDS3052C, Tektronix) with a self-trigger mode excited by neutrons from ${ }^{252} \mathrm{Cf}$. This setup is also described in detail in ref. 11.

\section{Results and Discussion}

Figure 1 shows the PL and PLE spectra of APLF80+Ce with $0.5 \mathrm{~mol} \%$ doping concentration. The fluorescence from the $5 \mathrm{~d}-4 \mathrm{f}$ transitions in Ce ions is clearly observed at $340 \mathrm{~nm}$ for all doping concentrations. As the Ce concentration increases, the fluorescence peak position shifts to longer wavelengths (Fig. 2). The X-ray induced fluorescence and PL spectra of the APLF80+Ce with doping concentrations ranging from 0.1

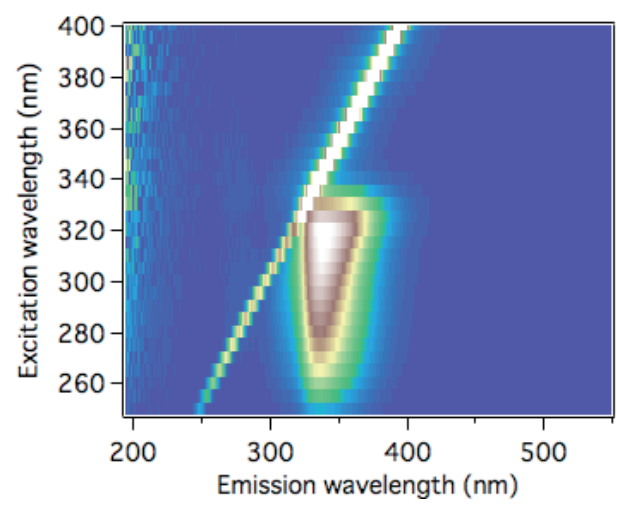

Fig. 1. (Color online) PL and PLE spectra of APLF80+Ce with 0.5 mol\% doping concentration.

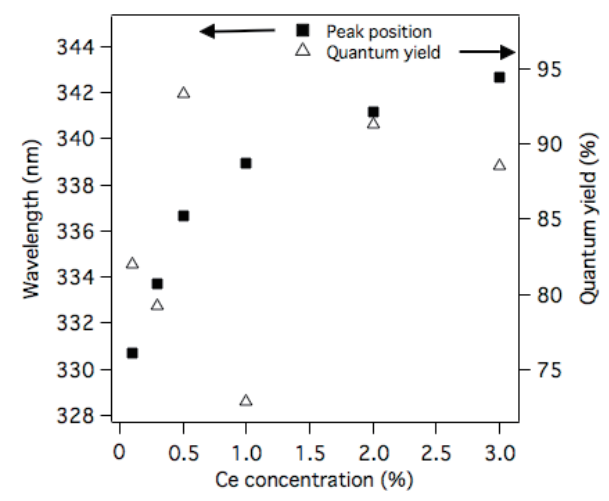

Fig. 2. Fluorescence peak position and quantum yield of APLF80+Ce with different doping concentrations. 
to $3 \mathrm{~mol} \%$ are shown in Fig. 3. In terms of the peak wavelength, both the X-ray induced fluorescence and PL are similar, but in terms of the intensities with increasing doping concentration, the two spectra are different. The fluorescence intensity of the samples with X-ray excitation increases with increasing Ce doping, while the fluorescence intensity with UV excitation is almost similar for different doping concentrations. The doping concentration dependence of the fluorescence intensity is not observed in the PL spectra because of the similar absorption coefficients at $260 \mathrm{~nm}\left(2.33 \pm 0.06 \mathrm{~cm}^{-1}\right)$ for all of the APLF80+Ce samples as shown in Fig. 4.

On the other hand, the quantum efficiency of the UV fluorescence is over $90 \%$ (Fig. 2). The $0.5 \mathrm{~mol} \%$ APLF $80+\mathrm{Ce}$ shows the highest quantum efficiency of $95 \%$. It is well known that trivalent $\mathrm{Ce}$ ion has strong UV luminescence. This is attributed to the electronic transition from the $5 \mathrm{~d}$ state to the $4 \mathrm{f}$ state. Meanwhile, there is no luminescence of tetravalent $\mathrm{Ce}$ ions due to the lack of $4 \mathrm{f}$ electrons. The transition from the $5 \mathrm{~d}$ state to the $4 \mathrm{f}$ state is dominant since the Ce ions are stable as trivalent ions in the APLF glass. This quantum efficiency is high for fluorescent materials in the UV region. $\mathrm{Ce}: \mathrm{LiCAF}_{6}$ laser crystals, for example, have a quantum efficiency of $80 \%$. $^{(12)}$ Glass materials usually have lower quantum efficiencies than laser crystals.

Figure 5 shows the pulse height distributions of APLF $80+$ Ce with doping concentrations ranging from 0.1 to $3 \mathrm{~mol} \%$. The $3 \mathrm{~mol} \%$ Ce-doped APLF has the highest quantum yield of about 400 photons, which suggests no occurrence of concentration quenching. In the case of neutron excitation, the reaction

$$
6 \mathrm{Li}+\mathrm{n} \rightarrow \alpha+\mathrm{T}
$$

occurs creating alpha particles $(\alpha)$ and tritium $(\mathrm{T})$ to excite the Ce ions in the samples. ${ }^{(13)}$ The $3 \mathrm{~mol} \% \mathrm{Ce}$ doping concentration is still not enough to achieve saturation. A doping concentration higher than $3 \mathrm{~mol} \%$ might possibly increase the quantum yield, but doping APLF with higher concentrations is difficult owing to the unstable nature of the APLF

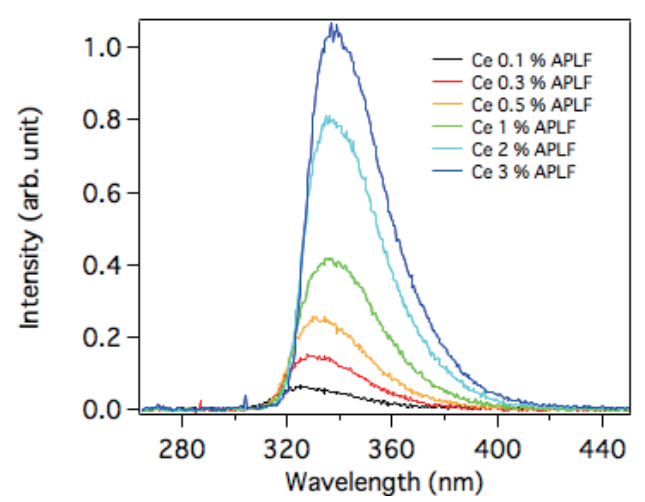

(a)

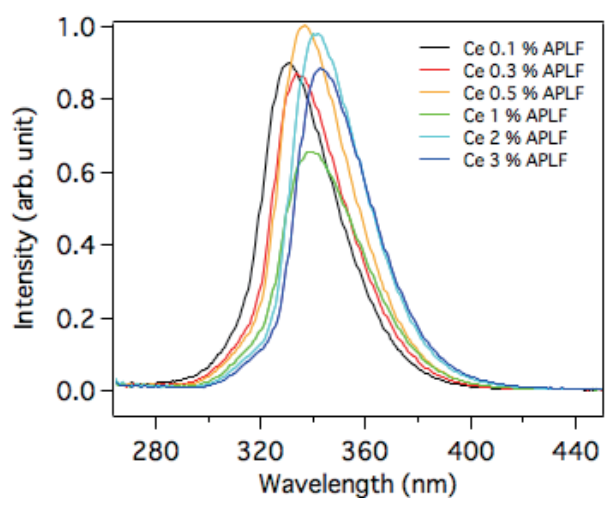

(b)

Fig. 3. (Color online) (a) X-ray induced fluorescence and (b) PL spectra of APLF80+Ce with doping concentrations. 


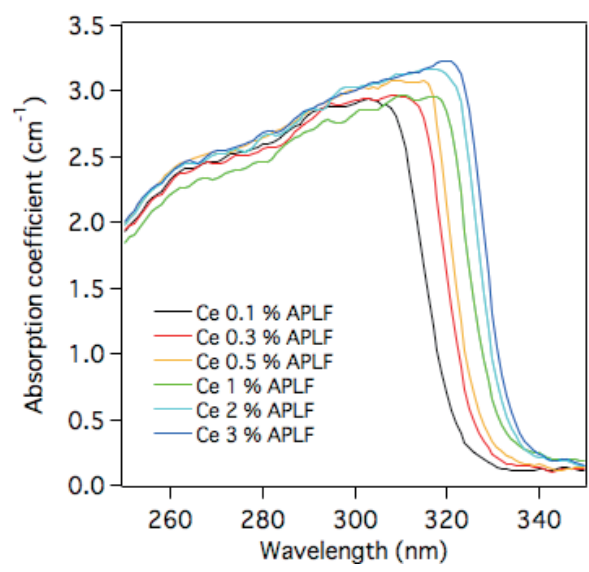

Fig. 4. (Color online) Absorption spectra of APLF80+Ce with different doping concentrations.

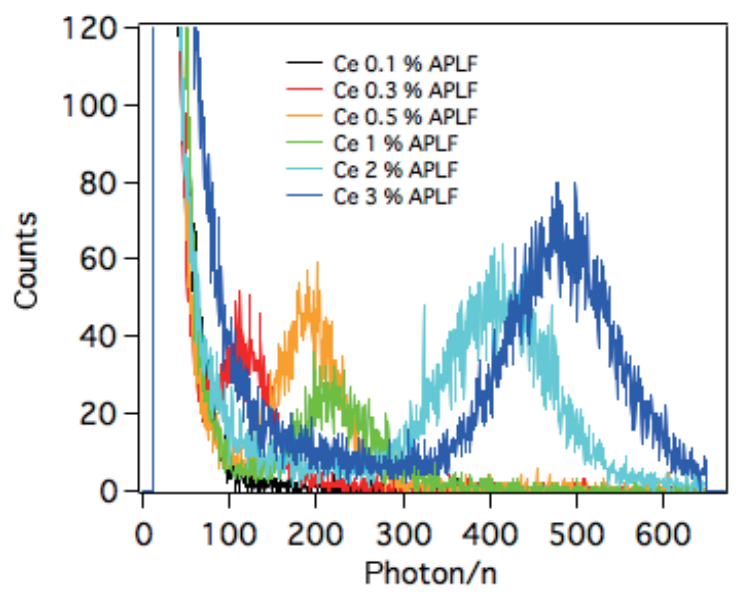

Fig. 5. (Color online) Pulse height distribution of APLF80+Ce with different doping concentrations and with ${ }^{252} \mathrm{Cf}$ excitation.

glass. The APLF samples contain about $80 \% \mathrm{LiF}$. As a well-known crystal with high transparency in the vacuum UV (VUV) region, LiF tends to crystalize easily. ${ }^{(14,15)}$ A Ce doping concentration more than $3 \mathrm{~mol} \%$ leads to an immediate crystallization during the cooling process. The scintillation yield was relatively low, but the PL efficiency was high. The excitation by particles includes various energy losses, such as those caused by defect trapping, phonon relaxation, and inelastic scattering, since glass materials are structurally inhomogeneous compared to crystals. Thermally or optically stimulated luminescence measurements are required to confirm the losses due to defects. ${ }^{(16)}$ Recently, it has been revealed that scintillation and storage-type luminescence have a complimentary relationship; ${ }^{(17)}$ thus, an efficient thermally or optically stimulated luminescence can be 


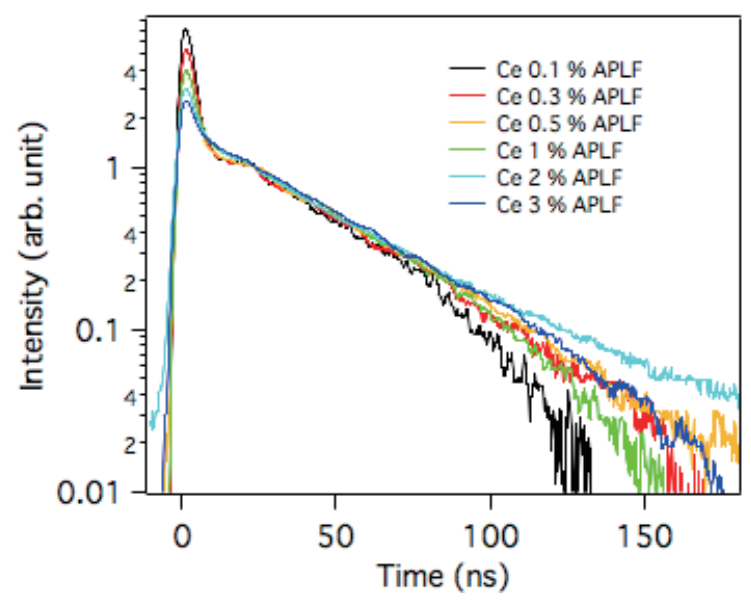

Fig. 6. (Color online) Decay time profiles of APLF80+Ce with different doping concentrations and with ${ }^{252} \mathrm{Cf}$ excitation.

expected in APLF glass. The decay times shown in Fig. 6 were constant in the range from 38 to $41 \mathrm{~ns}$ regardless of Ce concentration. The APLF80+Ce glasses have a greater advantage than a conventional Li glass scintillator because of a faster scintillation decay. ${ }^{(18)}$

\section{Conclusion}

Ce-rich APLF glass samples were successfully prepared by melt quenching. The optical properties of these samples used as scintillator and illuminator materials were dependent on the Ce doping concentration. The quantum efficiency of the $5 \mathrm{~d}-4 \mathrm{f}$ transition related to the $350 \mathrm{~nm}$ emission was over $95 \%$. These results suggest that trivalent $\mathrm{Ce}$ ions are incorporated into the APLF glass samples. The scintillation yield of around 400 photon $/ 252 \mathrm{Cf}$ with a decay time of 38 to $41 \mathrm{~ns}$ was observed without quenching due to high doping concentration. Ce-doped APLF glass holds promise as a potential UV illuminator and scintillator material.

\section{Acknowledgements}

This work was partially supported by the SPRITS program, Kyoto University, and the Cooperative Research Project of Research Institute of Electronics, Shizuoka University.

\section{References}

1 M. A. Dubinskii, V. V. Semashko, A. K. Naumov, R. Y. Abdulsabirov and S. L. Korableva: J. Mod. Opt. 40 (1993) 1.

2 M. Nikl: Phys. Status Solidi A 178 (2000) 595.

3 C. L. Melcher and J. S. Schweitzer: IEEE Trans. Nucl. Sci. 39 (1992) 502. 
4 K. Yamanoi, T. Murata, Y. Arikawa, T. Nakazato, M. Cadatal-Raduban, T. Shimizu, N. Sarukura, M. Nakai, T. Norimatsu, H. Nishimura, H. Azechi, S. Fujino, H. Yoshida, A. Yoshikawa, N. Satoh and H. Kan: Opt. Mater. 35 (2013) 1962.

5 Y. Arikawa, K. Yamanoi, T. Nakazato, E. S. Estacio, T. Shimizu, N. Sarukura, M. Nakai, T. Norimatsu, H. Azechi, T. Murata, S. Fujino, H. Yoshida, K. Kamada, Y. Usuki, T. Suyama, A. Yoshikawa, N. Sato and H. Kan: Rev. Sci. Inst. 80 (2009) 113504.

6 A. R. Spowart: Nucl. Instrum. Methods 140 (1977) 19.

7 E. J. Fairley and A. R. Spowart: Nucl. Instrum. Methods 150 (1978) 159.

8 Y. Arikawa, K. Yamanoi, T. Nagai, K. Watanabe, M. Kouno, K. Sakai, T. Nakazato, T. Shimizu, M. R. Cadatal, E. S. Estacio, N. Sarukura, M. Nakai, T. Norimatsu, H. Azechi, T. Murata, S. Fujino, H. Yoshida, N. Izumi, N. Satoh and H. Kan: Rev. Sci. Inst. 81 (2010) 106105.

9 T. Murata, Y. Arikawa, K. Watanabe, K. Yamanoi, M. Cadatal-Raduban, T. Nagai, M. Kouno, K. Sakai, T. Nakazato, T. Shimizu, N. Sarukura, M. Nakai, T. Norimatsu, H. Nishimura, H. Azechi, A. Yoshikawa, S. Fujino, H. Yoshida, N. Izumi, N. Sato and H. Kan: IEEE Trans. Nucl. Sci. 59 (2012) 2256.

10 T. Yanagida, K. Kamada, Y. Fujimoto, H. Yagi and T. Yanagitani: Opt. Mater. 35 (2013) 2480.

11 T. Yanagida, N. Kawaguchi, Y. Fujimoto, K. Fukuda, K. Watanabe, A. Yamazaki and A. Uritani: J. Lumin. 144 (2013) 212.

12 K. Shimamura, N. Mujilatu, K. Nakano, S. L. Baldochi, Z. Liu, H. Ohtake, N. Sarukura and T. Fukuda: J. Cryst. Growth 197 (1999) 896.

13 J. M. Neill, D. Huffman, C. A. Preskitt and J. C. Young: Nucl. Instrum. Methods 82 (1970) 162.

14 W. G. Johnston and J. J. Gilman: J. Appl. Phy. 30 (1959) 129.

15 D. C. Stockbarger: Rev. Sci. Inst. 7 (1936) 133.

16 D. D. Martino, A. Vedda, C. Montanari, E. Rosetta, E. Mihokova, M. Nik1, H. Sato, A. Yoshikawa and T. Fukuda: Opt. Mater. 30 (2007) 69.

17 T. Yanagida, Y. Fujimoto, K. Watanabe, K. Fukuda, N. Kawaguchi, Y. Miyamoto and H. Nanto: Radiat. Meas., DOI: 10.1016/j.radmeas.2014.03.020 (2014) (in press).

18 A. Nikitin, A. Fedorov and M. Korjik: IEEE Trans. Nucl. Sci. 60 (2013) 1044. 